\title{
Can regret prompt compensatory health behaviors? Findings from a clustered randomized trial with physically active adults
}

\begin{abstract}
BACKGROUND
Failure to resist temptation may make people experience an action-control emotion of regret. We explored whether evoking regret after participants' failure to attend physical exercise sessions would prompt them to undertake compensatory behaviors of different sorts.
\end{abstract}

\section{PARTICIPANTS AND PROCEDURE}

Physically active men and women $(N=133)$ were invited to attend a 6-session fitness course. Using cluster randomization, participants were assigned either to the experimental group with a regret-evoking message after they failed to show up at a fitness session $(n=69)$, or the control group, which did not receive the regret-evoking message $(n=64)$. After missing any of the first five fitness sessions participants reported whether they had engaged in compensatory health behaviors during the preceding 24 hours. Data were collected at pre-test, post-test, and after each missed fitness session.
RESULTS

Linear mixed-effects analysis showed no effects of the regret manipulation on compensatory behaviors such as physical activity, nutrition, or substance use avoidance. However, the manipulation resulted in lower levels of engagement in socializing behaviors.

\section{CONCLUSIONS}

The results suggest that people engage in compensatory behaviors after a lapse in physical exercise. Evoked regret may serve to a limited extent as a means to prompt compensatory behaviors.

\section{KEY WORDS}

compensatory health behaviors; regret; physical activity; clustered controlled trial

ORganization - 1: Faculty of Psychology, University of Social Sciences and Humanities SWPS, Wroclaw, Poland .

2: University of Colorado at Colorado Springs, Colorado Springs, USA - 3: Psychology Applied to Health,

University of Exeter Medical School, Exeter, United Kingdom

AUthors' CONTRibutions - A: Study design - B: Data collection - C: Statistical analysis - D: Data interpretation .

E: Manuscript preparation · F: Literature search · G: Funds collection

CORRESPonding AUTHOR - Katarzyna Byrka, Ph.D., University of Social Sciences and Humanities SWPS,

30 B Ostrowskiego Str., 53-238 Wrocław, Poland, e-mail: kbyrka@swps.edu.pl

TO CITE THIS ARTICLE - Byrka, K., Luszczynska, A., \& Abraham, C. (2018). Can regret prompt compensatory health

behaviors? Findings from a clustered randomized trial with physically active adults. Health Psychology Report, 6(4),

285-295. https://doi.org/10.5114/hpr.2018.77804

RECEIVED 12.03.2018 · REVIEWED 21.04.2018 · ACCEPTED 29.07.2018 · PUBLISHED 11.09.2018 


\section{BACKGROUND}

Failure to follow through on health-related intentions is often a consequence of giving into the temptations of everyday life. Such situations when a "weak will" (e.g., staying at home and watching a film) triumphs over long-term goals (e.g., becoming healthier through regular physical exercise) may evoke reflections on the outcome of one's own actions, and consequently lead to a cognitively-loaded and aversive emotion of regret (cf. Giner-Sorolla, 2001; Gilovich \& Medvec, 1995). Avoiding regret and managing motivational conflict between short-term temptations and long-term goals is foundational to self-regulation (Baumeister \& Heatherton, 1996; Baumeister, Stillwell, \& Heatherton, 1994, 1995).

According to cognitive dissonance theory (Festinger, 1957), one way to alleviate unpleasant emotions after giving in to temptation is changing cognitions concerning the consequences. Another option is to engage in behaviors that lead to the same goal as the non-performed behavior. According to the Compensatory Health Beliefs Model (CHBM; Rabiau, Knäuper, \& Miquelon, 2006), after failing to implement a health-related intention or after engaging in an unhealthy behavior individuals formulate compensatory beliefs. For example, individuals may believe that the harm caused by smoking cigarettes can be compensated for by drinking water (Knäuper, Rabiau, Cohen, \& Patriciu, 2004; Rabiau et al., 2006). So far, compensatory beliefs have attracted significant scholarly attention. With our research we aim to explore whether failures in pursuing an intended goal of being fit and healthy may prompt compensatory behaviors such as compensatory eating, exercising, or socializing. Moreover, because various inactions and omissions cause regret (Gilovich \& Medvec, 1995; Zeelenberg \& Pieters, 2007), we expect that its evocation will intensify the chances a goal is acted on. In other words, we predict that evoked regret may increase performance of compensatory health behaviors, especially when engaging in the target behavior is no longer possible (e.g., an opportunity to participate in a fitness session is no longer available). In particular, we extend existing research by testing whether prompting regret after failure to attend a fitness session increases the likelihood of self-reported engagement in various compensatory health behaviors.

\section{COMPENSATORY HEALTH BEHAVIORS}

In line with the literature, we define "compensatory behaviors" as intentional actions that are perceived as means of achieving distant goals such as being healthy, eating healthily, or being fit (Kronick, Auerbach, Stich, \& Knäuper, 2011; Rabiau et al., 2006). For example, a health-conscious individual who missed a fitness session may go jogging in a park or refrain from a calorie-rich snack.

Research to date has shown that when individuals are faced with a temptation that contradicts a distant goal they hold, they start to harbor compensatory cognitions, including compensatory intentions, in order to minimize unpleasant tension (Kronick \& Knäuper, 2010). Rabiau et al. (2006) posit that compensatory behaviors are means to the same healthrelated goal as a non-performed behavior and are preceded by compensatory health beliefs. This might occur, however, when individuals plan compensatory behaviors ahead or when they have higher levels of self-efficacy.

Recent studies have shown that compensatory behaviors are not necessarily positively linked to health beliefs. For example, research has showed that endorsing compensatory beliefs translates to a more calorie-loaded diet (Rabiau, Knäuper, Nguyen, Sufrategui, \& Polychronakos, 2009), weaker intentions to quit smoking (Radtke, Scholz, Keller, \& Hornung, 2012), or reduced likelihood of vaccination (Ernsting, Schwarzer, Lippke, \& Schneider, 2013).

Moreover, Kaklamanou, Armitage, and Jones (2013) showed in a think-aloud study that a distinction should be drawn between compensatory behaviors and compensatory beliefs. Radtke and Scholtz (2016), in turn, performed a more systematic study using self-reports in which they showed that compensatory health behaviors and compensatory health beliefs form two separate dimensions.

Compensatory cognitions may be sufficient in some situations to minimize emotional tension following failure to act upon intentions. However, if strong feelings of regret are evoked, then compensatory actions may occur.

\section{REGRET AS A TRIGGER OF ACTIONS}

The aversive emotion of regret motivates people to act in order to minimize its volume and strength (Gilovich \& Medvec, 1995). Not fulfilling one's goals is associated with breaching important personal norms (Janis \& Mann, 1977). This, in turn, produces feelings that something could have been done differently (Giner-Sorolla, 2001; Zeelenberg \& Pieters, 2007). Regret is a "cognitively-laden" emotion because reflection and reference to an alternative outcome are necessary to evoke it (Miller, Turnbull, \& McFarland, 1989). For example, individuals who missed a fitness class (session) would regret it if they recognized that attending the session would have contributed to their fitness, health, or well-being. In general, engaging in harmful actions (e.g., smoking) provokes more severe reactions of regret than inactions (e.g., missing a fitness session), but in the latter situation the emotion may be more persistent and last 
longer (Gilovich \& Medvec, 1995). The experience of regret is aversive to the extent that even hypothetical thinking about it motivates people to act in order to avoid the prospect of negative affect (Sheeran \& Orbell, 1999). For example, individuals who read scenarios in which the main character expressed regret after unsafe sex were more likely to delay sexual gratification (Quisenberry, Eddy, Patterson, Franck, \& Bickel, 2015). Greater anticipated regret, in turn, is associated with an increased likelihood of undertaking single and recurrent behaviors that are regretted when not performed, such as exercising (Abraham \& Sheeran, 2003, 2004), cancer pre-screening (de Nooijer, Lechner, Candel, \& de Vries, 2004), or safe sexual practices (Richard, de Vries, \& van der Pligt, 1998). Thoughts of regret have also been found to predict avoidance of healthimpairing behaviors such as smoking (Conner, Conner, Sandberg, McMillan, \& Higgins, 2006), self-harming (O’Connor \& Armitage, 2003), or drinking alcohol (Richard, van der Pligt, \& de Vries, 1996). A meta-analysis found a moderate relationship ( $r=.27$ ) between anticipated regret and health behavior (Sandberg \& Conner, 2008). Aside from a direct effect on behavior, regret has also been shown to moderate the relationship between intentions and behaviors (Abraham \& Sheeran, 2003). For example, adolescents' intentions to smoke were predictive for initiation of smoking only when they did not anticipate regret of doing so (Conner et al., 2006).

\section{RESEARCH GOALS}

Most research to date has explored the effects of regret manipulation with reference to a future perspective (e.g., anticipation of an affective state) and showed its effectiveness when a particular behavior could still be performed after the manipulation (e.g., Abraham \& Sheeran, 2003, 2004; de Nooijer et al., 2004; Richard et al., 1998). The majority of previous research evaluated the effects of manipulations evoking anticipated regret and relied on an imagined failure to act upon one's intentions, i.e., imagining a lapse followed by an imagined increase in negative emotions, such as regret (cf. Richard et al., 1998). Our study extends previous research and focuses on regret evoked post factum, that is, after a failure to actually act as intended.

To the best of our knowledge, no study so far has explored the effect of evoked regret on compensatory behaviors. The aim of this research was to examine whether regretting an opportunity to exercise would prompt compensatory health behaviors. In particular, we hypothesized that after missing a planned physical activity session, a regret-inducing text message and a regret-assessment item would increase the occurrence of compensatory health-promoting behaviors such as healthy nutrition, physical activ- ity, substance avoidance, socializing, and relaxing. We also expected that the effect of regret messages would be stronger over time, as opportunities to participate in a series of fitness sessions will decrease. Support for our hypotheses would demonstrate that evoking feelings of regret could serve as a means to motivate people to engage in healthy compensatory behaviors.

\section{PARTICIPANTS AND PROCEDURE}

\section{STUDY DESIGN}

We conducted a clustered randomized trial in which four of eight fitness courses were randomly assigned (using an internet-based randomizer) to the experimental condition in which regret was evoked and the other four to the control condition. As a consequence, 69 individuals received regret prompts (courses \#1, 2, 6, 8) while 64 did not (courses \#3, 4, 5, 7). Evoked regret was the independent variable, and self-reported compensatory health behaviors (physical activity, nutrition, substance avoidance, socializing and relaxation) were dependent variables (see Figure 1). Data were collected at seven points: at baseline $\left(t_{0}\right)$, at five post-fitness-session time points $\left(t_{1}-t_{5}\right)$ and, finally, at post-test, which was performed after the sixth session $\left(\mathrm{t}_{6}\right)$. For details see Figure 1. Gender was included as a covariate in all models, because research suggests that men and women differ in their engagement in various health-promoting behaviors (Anderson \& Bulik, 2004).

\section{PARTICIPANTS}

We recruited participants via the first author's university website, by personal approach in fitness centers, or by using a snowball procedure by asking participants who had volunteered to recommend the study to friends and acquaintances. All study participants were residents of the urban area of Wroclaw, a city in the south-west of Poland. Only individuals who were in good health and already engaged in moderate-to-vigorous physical activity (MVPA) for at least 150 minutes per week were included. The rationale for this criterion was that the fitness course offered to study participants required at least a moderate fitness level and capacity to perform MVPA for at least 60 minutes. Individuals who did not regularly perform MVPA could have been additionally affected by the physical challenge of performing MVPA during the fitness course.

Initially, 150 individuals meeting the inclusion criterion joined the study, of whom 133 (88.67\%) participated in at least one fitness session. These participants' mean age was $M=26.30(S D=6.98)$ ranging
Regret and compensatory behaviors 
$\mathbf{t}_{0}-$ At least a day before

$\mathbf{t}_{1}$ - Monday session after 20.00 or 21.00

Katarzyna Byrka, Aleksandra Luszczynska, Charles Abraham $\mathbf{t}_{\mathbf{2}}-$ Wednesday session after 20.00 or 21.00 $t_{3}$ - Friday session after 20.00 or 21.00

4 - Monday session after 20.00 or 21.00

$\mathbf{t}_{5}$ - Wednesday session after 20.00 or 21.00
Regret condition

courses \#1, 2, 6, 8

- Regret message

- Do you regret that you missed the session?

- Compensatory health behaviors

- Compensatory health behaviors

- Regret message

- Do you regret that you missed the session?

- Compensatory health behaviors

Regret condition

- Regret message

- Do you regret that you missed the session?

- Health behaviors

Regret condition

- Regret message

- Do you regret that you missed the session?

- Health behaviors

\begin{tabular}{l} 
Regret condition \\
\hline - Regret message \\
- Do you regret that you missed \\
the session? \\
- Compensatory health behaviors
\end{tabular}

Pre-test

(age, gender)

\author{
r)
}

intention

No regret condition

courses \#3, 4, 5, 7

- Message with a reminder to complete a diary

- Compensatory health behaviors

\section{No regret condition}

- Message with a reminder to complete a diary

- Compensatory health behaviors

$$
\text { No regret condition }
$$

- Message with a reminder to complete a diary

- Compensatory health behaviors

\section{No regret condition}

- Message with a reminder to complete a diary

- Compensatory health behaviors

\section{No regret condition}

- Message with a reminder to complete a diary

- Compensatory health behaviors

$\mathbf{t}_{6}$ - Friday session after 21.00 or 22.00

\section{Post-test measure}

Regret:

Do you regret that you missed any of the sessions?

Figure 1. Flowchart representing chronological stages of the study.

from 18 to 50 years. The vast majority were female $(82.71 \%, n=110)$. Men and women, however, were homogeneous with respect to age, $F(1,132)=0.62$, $p=.431$, education, $\chi^{2}(1, N=133)=2.29, p=.319$, and perceived socioeconomic status, $F(1,132)=0.23$, $p=.631$.

Participants either held a university degree (55.64\%) or had completed high school (43.61\%), apart from one person who reported upper primary school $(0.75 \%)$ as their highest level of educational attainment. On average, participants evaluated their perceived socioeconomic status as higher than that of the average family in Poland $(M=3.60, S D=0.87)$ when responding to a five-point scale ranging from below the status of an average family (1) to above the status of an average family (5). Most participants $(88.72 \%)$ had a normal body mass index (BMI), in the range $18-24$. Only $6(4.51 \%)$ participants scored below 18 and only $9(6.77 \%)$ reported weight and height indicative of BMI above 25 .

\section{PROCEDURE}

During online registration the participants received information about the study procedures, gave informed consent and confirmed that they were in good health. Next, they completed a baseline questionnaire 
$\left(\mathrm{t}_{0}\right)$ including socio-demographic variables as well as other questionnaires administered as part of a larger research project on compensatory behaviors. At this stage, participants also provided their e-mail addresses and phone numbers.

After completing the questionnaire, participants booked one of eight proposed fitness courses to suit their personal schedule. Each course lasted 12 days and consisted of six 60-minute fitness sessions conducted every two days. The courses were organized by a certified and registered fitness studio located in a well-known hotel in the city center, and were conducted by certified fitness instructors.

The sessions were based on the Functional Movement System approach. Exercises, including inline lunges, v-poses, deep squats, and T-stands, focused on movement patterns typical for everyday functioning and were demanding for a person of averages fitness. The courses were organized for the purpose of the reported research and for participants of the study only. The scenarios for the exercise sessions were the same for all eight groups. Participation in the course was free of charge. The number of registered participants was similar across all eight courses $(M=16.63, S D=2.67)$, ranging from 12 to 19 . Fitness instructors and research assistants were blind to the tested hypotheses.

Within an hour of the end of each fitness session from $1\left(t_{1}\right)$ to $5\left(t_{5}\right)$, all participants who had booked that session were contacted via text message and e-mail. The e-mail included a link to a brief questionnaire. Participants who had not attended a given fitness session were asked to report whether they had engaged in a health-promoting activity during the 24 hours before completing the questionnaire (at each of five time points, $t_{1}$ to $t_{5}$ ) instead of participating in a fitness session. ${ }^{1}$ After the first failure to attend, participants were asked to provide reports of their affective states (measured with items derived from I-PANAS-SF). After the sixth and final $\left(\mathrm{t}_{6}\right)$ session, post-test data were collected.

The regret manipulation was delivered after each missed fitness session. The manipulation included: 1) a text message sent via mobile phone and 2) a questionnaire item assessing regret, adapted from the study by Sheeran and Orbell (1999) on anticipated regret. The text message read as follows: Regularity of exercise is important for keeping in shape. Perhaps you regret that you missed your last training session. Please complete a questionnaire that will be sent to you via $e$-mail. The text message was followed by an e-mail with a link to the questionnaire on compensatory behaviors. For participants who received the regret text message, the first item of the subsequent questionnaire asked whether they regretted missing a fitness session, offering five response options from 1 ( $I$ do not regret it at all) to 5 (I regret it a lot). Items assessing compensatory behaviors followed.
The text message in the control condition was as follows: Regularity of exercise is important for keeping in shape. Please complete a questionnaire that will be sent to you via e-mail. To avoid mere measurement effects (Sherman, 1980), the post-session questionnaire for control groups did not contain the regret assessment item. If a participant was present at a given fitness session, they received a form containing activities that have negative health consequences, but the related results are beyond the scope of this text.

Participants in the experimental and control conditions who failed to complete the questionnaire received a text reminder during the following day. As the courses took place every two days, participants could respond to post-session questionnaires not later than 24 hours after a given session.

As compensation for their efforts, each participant received a thermal cup worth roughly $15 €(£ 10)$. They were also provided with further details about the research objective. Data were collected between February and April 2015 in Wroclaw, Poland. The study was approved by the Internal Ethics Review Board at the first author's institution.

\section{MEASURES}

Compensatory behaviors were measured at $t_{1}, t_{2}, t_{3}$, $t_{4}$ and $t_{5}$. Affective states were measured as control variables after a participant missed a session for the first time. Therefore, measurement of affective states could take place after any of the five fitness sessions $\left(\mathrm{t}_{1}-\mathrm{t}_{5}\right)$. Regret was measured at post-test $\left(\mathrm{t}_{6}\right)$.

Compensatory behaviors. Knäuper et al. (2004) identified four health subdomains in which compensatory beliefs are formed: substance use, eating/ sleeping habits, stress, and weight regulation. The present study applies a similar approach and treats compensatory behaviors as belonging to various health subdomains rather than forming a one-dimensional construct. Accordingly, we measured five types of behavior that could serve as compensatory actions following a missed fitness session: physical activity, eating, substance avoidance, relaxation and socializing. We opted for including relaxation and socializing in line with recent taxonomies of health behaviors, which contain a broad spectrum of behaviors that directly refer to psychosocial health and general well-being through stress reduction and maintenance of relationships (Byrka \& Kaiser, 2013; Nudelman \& Shiloh, 2015).

Compensatory physical activity was measured with three statements from the Godin-Shephard Leisure-Time Exercise Questionnaire (Godin, 2011). "Indicate whether in the last 24 hours you performed strenuous exercise (heart beats rapidly, e.g., running, jogging, hockey, football, soccer, squash, basketball,
Regret and compensatory behaviors 
Katarzyna Byrka,

Aleksandra

Luszczynska,

Charles Abraham cross country skiing, judo, roller skating, vigorous swimming, vigorous long distance bicycling)"; "Indicate whether in the last 24 hours you performed moderate exercise (not exhausting; e.g., fast walking, baseball, tennis, easy bicycling, volleyball, badminton, easy swimming, alpine skiing, popular and folk dancing)"; "Indicate whether in the last 24 hours you performed mild exercise (minimal effort, e.g., yoga, archery, fishing from river bank, bowling, horseshoes, golf, snow-mobiling, easy walking)" and with one question adapted from the General Health Performance (GHP) scale by Byrka and Kaiser (2013; for the rationale see Kaiser, Byrka, \& Hartig, 2010). Participants chose one of four options in response to each question: disagree (1), partially disagree (2), partially agree (3) and agree (4), indicating whether they had engaged in physical activity of high, moderate, or mild intensity and whether they had chosen walking or using public transportation instead of driving in the previous 24 hours. The same response format was used for all compensatory behaviors. Internal consistency of the compensatory physical activity was Cronbach's $\alpha \mathrm{t}_{1}=.61, \mathrm{t}_{2}=.62, \mathrm{t}_{3}=.49, \mathrm{t}_{4}=.63$, $\mathrm{t}_{5}=.61$ (as alpha coefficient depends on the length of a scale, values below .75 are expected in short scales; see Cortina, 1993). Compensatory nutrition was measured with four questions adapted from the GHP scale: "I avoided fast food and semi-prepared meals, I avoided sweets (cakes, cookies, chocolate), I took an extra helping of vegetables, I had an extra serving of fruit". Internal consistency of the scale was Cronbach's $\alpha \mathrm{t}_{1}=.53, \mathrm{t}_{2}=.56, \mathrm{t}_{3}=.75, \mathrm{t}_{4}=.33, \mathrm{t}_{5}=.69$. Compensatory substance avoidance was measured with a single item, "I refrained from coffee, cigarettes, or alcohol". Compensatory socializing was measured with one item, "I spent this time meeting friends and colleagues". Compensatory relaxation was measured with a single item, "I spent time on relaxation (e.g., reading a book, walking in a park)".

Affective states. Positive and negative affect was measured with the Polish version of the I-PANAS-SF scale developed by Thompson (2007). The scale consists of 10 items that are responded to on a 5-point response scale ranging from 1 (not at all) to 5 (a lot) indicating how a person feels at a given moment. Items that measured positive affect were active, alert, attentive, determined and inspired. Items for a negative affect were afraid, ashamed, hostile, nervous and upset. The mean for five items representing positive and negative affective states was used in this study, with $M_{\text {pos }}=3.20\left(S D_{\text {pos }}=0.64\right)$ for the positive affect scale and $M_{\text {neg }}=1.83\left(S D_{n e g}=0.66\right)$ for the negative affect scale. Internal consistency of both subscales was Cronbach's $\alpha=.75$.

Regret. At the post-test measure $\left(\mathrm{t}_{6}\right)$ regret was measured in experimental and control questionnaires with one question adopted from Sheeran and Orbell (1999), "To what extent did you perceive regret if you missed a fitness session?" Responses ranged from 1 (not at all) to 5 ( a lot), with a non-applicable option. The mean item score was high: $M=4.16(S D=0.93)$.

\section{RESULTS}

We present the results in the following sequence. First, we compare participants who dropped out from the study with those who completed the procedure to the end. Second, we present the randomization check and the manipulation check for the regret and control conditions. Finally, we test the effect of the regret manipulation on compensatory health behaviors from five subdomains. We chose linear-mixed effects analysis as our statistical approach to account for within-participants variance. Due to sample-size restrictions we did not run multilevel analysis with particular courses as clusters.

\section{ATTRITION}

Participants who dropped out $(n=17,11.33 \%)$ and those who attended at least one fitness session did not differ in age, $F(1,149)=0.07, p=.800$, gender, $\chi^{2}(1, N=150)=0.40, p=.529$, education, $\chi^{2}(1, N=150)=3.02, p=.221$, or income, $F(1,149)=2.65$, $p=.106$. We defined a dropout as a situation in which a participant completed a $t_{0}$ questionnaire, but failed to appear at any of the fitness sessions.

\section{RANDOMIZATION CHECK}

Participants who received the regret manipulation $(n=69)$ and those who did not in the control condition $(n=64)$ did not differ in gender, $\chi^{2}(1, N=133)=0.00$, $p=.975$, education, $\chi^{2}(1, N=133)=1.03, p=.598$, or income, $F(1,132)=1.20, p=.274$. The groups differed in age, $F(1,132)=6.34, p=.013$. Participants in the experimental condition were older $(M=27.74$, $S D=7.60)$ than those in the control condition $(M=24.75, S D=5.92)$.

\section{MANIPULATION CHECK}

In line with previous research inducing specific negative emotions (e.g., fear of death, anticipated regret), we did not use a manipulation check directly targeting manipulated emotion (cf. Abraham \& Sheeran, 2003, 2004; Hayes, Schimel, Arndt, \& Faucher, 2010), because such a manipulation check might have evoked the respective emotion. Instead, in line with previous research manipulating through the use of specific negative emotions (e.g., fear of death; Hayes et al., 2010), we measured the immediate influence 
of the manipulation on a global index of negative affect. This was done to exclude the possibility that the manipulation would result in a global change of negative affect (i.e., not regret-specific), which would be indicative of a shift in negative affect (not regret solely). Thus, we analyzed the effect of the manipulation on the general level of negative affect of the participants after they missed a session for the first time, and the one-way ANOVA revealed no significant effect, $F(1,87)=2.60, p=.111$. Thus, the effects of the manipulation cannot be ascribed solely to an increase of overall negative affect. Additionally, we tested for long-term effects of the manipulation with regret, asking participants at $\mathrm{t}_{6}$ (for some participants from 2 to 10 days after the last text message with the regret manipulation) whether they had felt regret after missing any of the fitness sessions. Although not significant, the effect was in the expected direction, with participants who had received the manipulation reporting higher regret in the post-test $(M=4.23$, $S D=0.89)$ than participants in the control condition $(M=4.08, S D=0.98)$, Hedge's $g=0.16(95 \%$ CI $[-0.04$, $0.36])$.

Additionally, we calculated means of participants' responses on the regret-assessment item. It was used as a manipulation of regret, and therefore the relevant data are available from the experimental group only. One noteworthy result is that reported regret was above 3 on a response scale ranging from 1 to $5\left[M_{t 1}=3.93\left(S D_{t 1}=0.80\right), M_{t 2}=3.64\left(S D_{t 2}=1.12\right)\right.$, $M_{t 3}=3.75\left(S D_{t 3}=0.97\right), M_{t 4}=4.00\left(S D_{t 4}=0.92\right)$, $\left.M_{t 5}=3.96\left(S D_{t 5}=0.98\right)\right]$.

\section{EFFECTS OF REGRET ON COMPENSATORY BEHAVIORS}

We performed five linear mixed-effects analyses (employing a compound symmetry option to model the variance structure) to test the effects of regret on compensatory behaviors in the domains of physical activity, nutrition, substance avoidance, socializing and relaxation. In all analyses, the independent variables were the regret manipulation and the time of measurement as a repeated variable.

In the model of compensatory physical activity, no statistically significant main effects of regret $(F(1,92.63)=2.05, p=.312$, time of measurement, $F(4,119.41)=0.88, p=.478)$ or interactions of these two variables $(F(4,119.22)=0.51, p=.729)$ were observed.

Neither the effects of regret, $F(1,94.87)=0.00$, $p=.957$, time of measurement, $F(4,138.02)=0.91$, $p=.458$, nor interactions of these two variables, $F(4,137.79)=0.60, p=.661$, were significant in the case of the model with nutrition as a dependent variable.

In the model with compensatory substance avoidance, the regret manipulation had no significant effect, $F(1,95.55)=0.01, p=.921$. The effect of time fell short of the conventional significance level, but it was in the predicted direction $F(4,122.19)=2.05$, $p=.091$. As the course progressed, participants became more eager to compensate for their absence at a session by abstaining from substances such as coffee, cigarettes, or alcohol. The interaction effect of regret and time on compensatory substance avoidance was not significant, $F(4,122.00)=1.21, p=.312$. Pseudo- $R^{2}$ for the whole model equals .06 .

The analysis yielded a significant main effect of regret on socializing, $F(1,92.13)=8.95, p=.004$. Participants receiving the regret manipulation reported significantly less socializing $(M=3.01, S E=0.17$, $95 \% \mathrm{CI}[2.68,3.35])$ with friends and colleagues after missing a fitness session than participants in the control condition $(M=2.34, S E=0.15,95 \% \mathrm{CI}[2.01,2.64])$. The main effect of time of measurement was insignificant, $F(4,134.44)=1.93, p=.109$. The interaction of regret and time was significant $F(4,134.20)=2.74$, $p=.031$ (see Figure 2). Pseudo- $R^{2}$ for the whole model equals .15.

In the case of compensatory relaxation we did not find a statistically significant main effect of regret at the conventional level of $p<.05, F(1,95.66)=3.05$, $p=.084$. Still, the results were in the same direction as with socializing. Compared to controls $(M=3.08$, $S E=0.17,95 \% \mathrm{CI}[2.74,3.42])$ experimental group participants engaged less frequently $(M=2.68, S E=0.15$, $95 \% \mathrm{CI}[2.37,2.98])$ in such compensatory behaviors as relaxing through reading a book or going for a walk. The effect of time of measurement, $F(4,123.22)=0.67$, $p=.612$, and the interaction of regret and time were not significant, $F(4,123.03)=7.44, p=.564$. Pseudo- $R^{2}$ for the whole model equals .09 .

Gender was a significant covariate only in the model in which nutrition was a dependent variable,

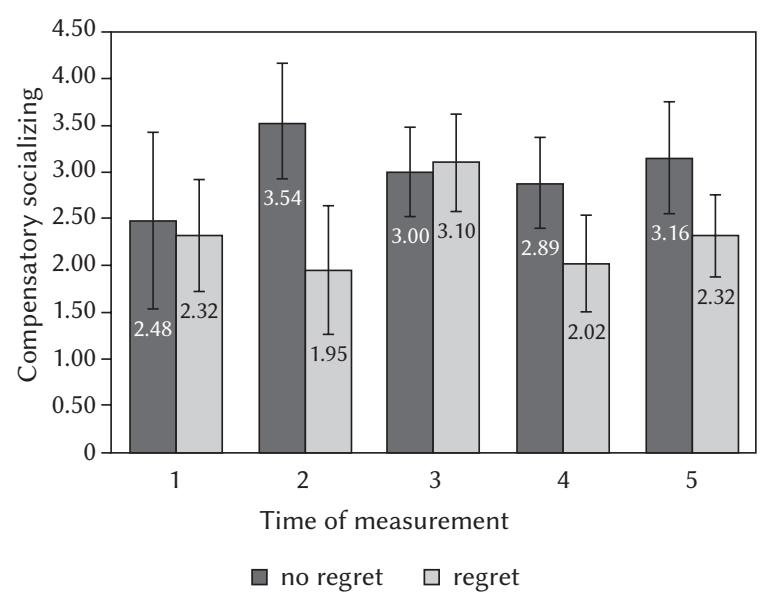

Figure 2. Compensatory socializing as a function of regret manipulation and time of measurement. Note. Means are adjusted for gender. Vertical bars represent $95 \%$ confidence.
Regret and compensatory behaviors 
Table 1

Sub-sample sizes, estimated marginal means, standard errors, confidence intervals of compensatory behavior in the control and the regret conditions

Katarzyna Byrka, Aleksandra Luszczynska, Charles Abraham

\begin{tabular}{|c|c|c|c|c|c|c|c|c|c|c|c|}
\hline \multirow{2}{*}{$\begin{array}{l}\text { Compensatory } \\
\text { behaviors }\end{array}$} & & \multicolumn{5}{|c|}{ Control condition } & \multicolumn{5}{|c|}{ Regret condition } \\
\hline & & $\mathrm{t}_{1}$ & $\mathrm{t}_{2}$ & $\mathrm{t}_{3}$ & $\mathrm{t}_{4}$ & $\mathrm{t}_{5}$ & $\mathrm{t}_{1}$ & $t_{2}$ & $\mathrm{t}_{3}$ & $\mathrm{t}_{4}$ & $\mathrm{t}_{5}$ \\
\hline \multirow{5}{*}{$\begin{array}{l}\text { Compensatory } \\
\text { physical activity }\end{array}$} & $n$ & 6 & 14 & 24 & 22 & 15 & 15 & 11 & 20 & 20 & 28 \\
\hline & $M$ & 2.51 & 2.75 & 2.40 & 2.66 & 2.44 & 2.13 & 2.59 & 2.49 & 2.39 & 2.32 \\
\hline & $S E$ & 0.32 & 0.21 & 0.17 & 0.17 & 0.21 & 0.21 & 0.23 & 0.18 & 0.18 & 0.16 \\
\hline & $95 \% \mathrm{Cl}_{\text {lower }}$ & 1.87 & 2.33 & 2.06 & 2.32 & 2.04 & 1.72 & 2.12 & 2.14 & 2.03 & 2.01 \\
\hline & $95 \% \mathrm{Cl}_{\text {upper }}$ & 3.14 & 3.17 & 2.73 & 3.01 & 2.85 & 2.53 & 3.05 & 2.85 & 2.74 & 2.62 \\
\hline \multirow{4}{*}{$\begin{array}{l}\text { Compensatory } \\
\text { nutrition }\end{array}$} & M & 3.14 & 3.07 & 2.82 & 3.29 & 3.04 & 2.95 & 2.98 & 3.09 & 3.18 & 3.12 \\
\hline & $S E$ & 0.30 & 0.20 & 0.15 & 0.16 & 0.19 & 0.19 & 0.22 & 0.17 & 0.17 & 0.14 \\
\hline & $95 \% \mathrm{Cl}_{\text {lower }}$ & 2.55 & 2.68 & 2.52 & 2.98 & 2.66 & 2.57 & 2.54 & 2.77 & 2.86 & 2.84 \\
\hline & $95 \% \mathrm{Cl}_{\text {upper }}$ & 3.73 & 3.45 & 3.12 & 3.59 & 3.41 & 3.32 & 3.41 & 3.42 & 3.51 & 3.39 \\
\hline \multirow{4}{*}{$\begin{array}{l}\text { Compensatory } \\
\text { substance avoidance }\end{array}$} & M & 3.24 & 2.92 & 2.92 & 3.18 & 2.73 & 3.20 & 3.40 & 2.37 & 3.03 & 2.88 \\
\hline & $S E$ & 0.45 & 0.29 & 0.23 & 0.24 & 0.29 & 0.29 & 0.33 & 0.25 & 0.25 & 0.22 \\
\hline & $95 \% \mathrm{Cl}_{\text {lower }}$ & 2.36 & 2.34 & 2.46 & 2.71 & 2.17 & 2.63 & 2.75 & 1.88 & 2.54 & 2.45 \\
\hline & $95 \% \mathrm{Cl}_{\text {upper }}$ & 4.12 & 3.50 & 3.38 & 3.64 & 3.30 & 3.77 & 4.04 & 2.86 & 3.52 & 3.30 \\
\hline \multirow{4}{*}{$\begin{array}{l}\text { Compensatory } \\
\text { socializing }\end{array}$} & M & 2.48 & 3.50 & 3.00 & 2.89 & 3.16 & 2.32 & 1.95 & 3.10 & 2.02 & 2.32 \\
\hline & $S E$ & 0.48 & 0.31 & 0.24 & 0.25 & 0.30 & 0.30 & 0.35 & 0.26 & 0.26 & 0.23 \\
\hline & $95 \% \mathrm{Cl}_{\text {lower }}$ & 1.54 & 2.92 & 2.52 & 2.40 & 2.56 & 1.72 & 1.26 & 2.58 & 1.50 & 1.87 \\
\hline & $95 \% \mathrm{Cl}_{\text {upper }}$ & 3.43 & 4.16 & 3.48 & 3.37 & 3.75 & 2.93 & 2.64 & 3.62 & 2.54 & 2.76 \\
\hline \multirow{4}{*}{$\begin{array}{l}\text { Compensatory } \\
\text { relaxation }\end{array}$} & M & 3.39 & 2.72 & 2.96 & 3.22 & 3.13 & 2.33 & 2.55 & 2.81 & 2.76 & 2.95 \\
\hline & $S E$ & 0.45 & 0.30 & 0.24 & 0.24 & 0.29 & 0.29 & 0.33 & 0.25 & 0.25 & 0.22 \\
\hline & $95 \% \mathrm{Cl}_{\text {lower }}$ & 2.50 & 2.13 & 2.49 & 2.75 & 2.56 & 1.75 & 1.90 & 2.32 & 2.26 & 2.52 \\
\hline & $95 \% \mathrm{Cl}_{\text {upper }}$ & 4.29 & 3.31 & 3.42 & 3.69 & 3.70 & 2.90 & 3.21 & 3.31 & 3.26 & 3.38 \\
\hline
\end{tabular}

$F(1,93.50)=4.51, p=.036$. Descriptive statistics for all measurements of compensatory behaviors are given in Table 1.

\section{ADDITIONAL CONTROL VARIABLES}

Aside from gender, we examined the effect of other intuitive covariates, i.e., age, regret measured in posttest, and the attendance at the next sessions. No significant effects were found after controlling for these variables.

\section{DISCUSSION}

In this study we explored the effects of evoked regret on performance of compensatory behaviors after a failure to engage in an intended health-promoting behavior, namely, attending a fitness training session. The regret-evoking manipulation following missed fitness sessions did not affect compensatory behaviors in any predicted domains. The manipulation had no effect on physical activity (e.g., exercising, walking). Based on previous findings on the influence of anticipated regret on target behavior (Abraham \& Sheeran, 2003; de Nooijer et al., 2004; Richard et al., 1998), such effects could have been explained by reference to differences in types of behaviors. In the anticipated regret paradigm, the regretted behavior and the target behavior are virtually the same. For example, a person is asked to evaluate anticipated regret of not exercising, and then self-reported or overt exercising is measured. We show that in situations where the individual is not able to engage at once in the missed behavior, it is possible that regret may not lead to engaging in a behavior which is of a highly similar form but is not exactly the same. For 
example, a lapse in one type of physical activity may not necessarily lead to replacement of that behavior with engagement in another type of physical activity.

Two physical activity behaviors, such as participation in a group fitness session supervised by a popular coach in an attractive location and jogging alone in a park, may be similar in one dimension, such as caloric expenditure. However, engaging in these two different types of physical activity may satisfy different needs and motives related to social recognition, stress management, endurance, positive health, challenge, enjoyment, etc., (Kilpatrick, Hebert, \& Bartolomew, 2005). Therefore, two types of activities may be perceived as too distinct to adequately compensate for one another. Future research should explore whether similarity of behaviors plays a role in the compensation process.

The regret-evoking manipulation did not affect compensatory nutrition. It seems that variables other than regret may have a stronger effect on compensatory eating or moderate the effects of regret on compensatory eating. For example, gender was a significant covariate in our study and women reported more compensatory nutrition behaviors after missing a training session than did men. The role of gender in compensatory eating patterns in clinical groups has been established in previous research (see e.g., Anderson \& Bulik, 2004) and probably played an important role in our study as well.

Evoked regret affected socializing with others. In particular, participants who received the regret manipulation after missing a fitness session reported less socializing with friends than did participants in the control condition. This finding is somewhat surprising and contrary to our predictions. Participating in a fitness session may be perceived as an activity which, aside from supporting weight management and good physical health, prompts pleasurable social interactions, relaxation and stress management (Kilpatrick et al., 2005). Socializing with friends may satisfy those non-physical needs as well. Therefore, we expected that the lapse in exercise would be compensated for by spending time with friends. Nonetheless, pleasurable and relaxing social activities may be perceived as behaviors triggering unhealthy diet, overeating and consumption of "forbidden" food (Carels et al., 2001). In sum, socializing with friends was likely perceived as leading to opposite effects in terms of weight management than physical activity. Therefore, withdrawing from such behaviors as socializing may present the best fit as a compensatory behavior for missing a fitness training session. Individuals who were exposed to the regret-evoking manipulation might have aimed at achieving balance in terms of caloric intake and expenditure. Another noteworthy result is that while the effects of regret on compensatory relaxation did not satisfy the conventional significance test criterion (see Table 1), they were consistent with findings on compensatory socializing. That is to say, participants who received the regret manipulation reported spending less time on relaxing (e.g., reading a book, strolling in a park) than participants in the control condition.

Non-significant results in the case of physical exercise, nutrition and substance avoidance may be also explained by reference to the subtlety of the regret manipulation. It is possible that the regret evoked by the text message and the regret-assessment question was not strong enough to prompt compensatory behaviors in the mentioned domains. It is always possible that participants might not have read the text messages with much attention. Nonetheless, ineffectiveness of the regret-assessment item manipulation would have been more surprising as it has been validated in previous research on anticipated regret (see Sheeran \& Orbell, 1999). Moreover, in the study design used here, most participants received multiple messages as they missed sessions more than once. It is possible that compensation which involves engagement in behaviors of different forms (e.g., exercising is compensated for by avoiding sweets) requires stronger and long-lasting feelings of regret. Furthermore, when the opportunity to engage in the target behavior is missed, people have a whole spectrum of possible compensatory behaviors to engage in. We measured only some of these potential behaviors.

The results might also have been affected by the fact that the physical exercise was planned by us rather than by the participants. Zeelenberg and Pieters (2007) point to agency and the opportunity to choose between alternatives as prerequisites of regret. Future research will show whether similar effects can be observed when health-promoting activities are spontaneously undertaken by participants rather than pre-planned by a research team.

Our research extends the literature on compensatory reactions engaged in by people following failure to resist a temptation. Much research so far has concentrated on compensatory health beliefs as means of alleviating the motivational conflict (Knäuper et al., 2004; Rabiau et al., 2006; Miquelon, Knäuper, \& Vallerand, 2012). The present study goes beyond these findings. We have demonstrated that evoked regret prompts individuals to undertake some types of compensatory behaviors after they missed planned exercise sessions. In particular, it appears that individuals refrain from behaviors such as compensatory socializing, which may satisfy needs across several psychosocial dimensions. Socializing may serve social recognition and stress management, but may play an opposite role in weight management, as the company of others has previously been found to prompt overeating (Carels et al., 2001).

This study is the first account of the effects of regret on compensatory behaviors after failure to
Regret and compensatory behaviors 
Katarzyna Byrka,

Aleksandra

Luszczynska,

Charles Abraham achieve a goal to improve health. This paradigm is worth further exploration in the broader context of various types of missed opportunities to engage in health-promoting behaviors. Future research should also explore the role of compensatory health beliefs and of other emotions such as guilt and shame in the process of regulating the tension that arises from giving in to temptations.

This work was financially supported by a grant 2014/13/D/HS6/01423 from the National Science Center, Poland. The contribution of Aleksandra Luszczynska was supported by BST/Wroc/2016 funds from Ministry of Science and Higher Education, Poland. The contribution of Charles Abraham was supported by the UK National Institute for Health Research (NIHR) Collaboration for Leadership in Applied Health Research and Care of the South West Peninsula (PenCLAHRC) but the views expressed in this paper are those of the authors and not necessarily those of NIHR or the UK Department of Health. The authors thank Magdalena Dubas, Wojciech Mitek and Renata Pietraszek for their contribution to data collection.

\section{ENDNOTE}

1 On average, participants attended more than three out of five fitness sessions after which regret manipulations were used, $M=3.48(S D=1.36)$. Only $29.32 \%$ $(n=39)$ attended all five analyzed sessions, $26.32 \%$ ( $n=35)$ missed one, $20.30 \%(n=27)$ two, $11.28 \%$ $(n=15)$ three, and $12.78 \%(n=17)$ four sessions.

\section{REFERENCES}

Abraham, C., \& Sheeran, P. (2003). Acting on intentions: The role of anticipated regret. British Journal of Social Psychology, 42, 495-511. https://doi. org/10.1348/014466603322595248

Abraham, C., \& Sheeran, P. (2004). Deciding to exercise: The role of anticipated regret. British Journal of Health Psychology, 9, 269-278. https://doi. org/10.1348/135910704773891096

Anderson, C. B., \& Bulik, C. M. (2004). Gender differences in compensatory behaviours, weight and shape salience, and drive for thinness. Eating Behaviours, 5, 1-11. https://doi.org/10.1016/j.eatbeh.2003.07.001

Baumeister, R. F., \& Heatherton, T. F. (1996). Self-regulation failure: An overview. Psychological Inquiry, 7, 1-15.

Baumeister, R. F., Stillwell, A. M., \& Heatherton, T. F. (1994). Guilt: An interpersonal approach. Psychological Bulletin, 115, 243-267. https://doi. org/10.1037/0033-2909.115.2.243

Baumeister, R. F., Stillwell, A. M., \& Heatherton, T. F. (1995). Personal narratives about guilt: Role in ac- tion control and interpersonal relationships. Basic and Applied Social Psychology, 17, 173-198. https:// doi.org/10.1080/01973533.1995.9646138

Byrka, K., \& Kaiser, F. G. (2013). Health performance of individuals within the Campbell paradigm. International Journal of Psychology, 48, 986-999. https://doi.org/10.1080/00207594.2012.702215

Carels, R. A., Hoffman, J., Collins, A., Raber, A. C., Cacciapaglia, H., \& O’Brien, W. H. (2001). Ecological momentary assessment of temptation and lapse in dieting. Eating Behaviours, 2, 307-321. https://doi.org/10.1016/S1471-0153(01)00037-X

Conner, M., Conner, M., Sandberg, T., McMillan, B., \& Higgins, A. (2006). Role of anticipated regret, intentions and intention stability in adolescent smoking initiation. British Journal of Health Psychology, 11, 85-101. https://doi.org/10.1348/135910705X40997

Cortina, J. M. (1993). What is coefficient alpha? An examination of theory and applications. Journal of Applied Psychology, 78, 98-104.

de Nooijer, J., Lechner, L., Candel, M., \& de Vries, H. (2004). Short- and long-term effects of tailored information versus general information on determinants and intentions related to early detection of cancer. Preventive Medicine, 38, 694-703. https:// doi.org/10.1016/j.ypmed.2003.12.016

Ernsting, A., Schwarzer, R., Lippke, S., \& Schneider, M. (2013). 'I do not need a flu shot because I lead a healthy lifestyle': Compensatory health beliefs make vaccination less likely. Journal of Health Psychology, 18, 825-836.

Festinger, L. (1957). A theory of cognitive dissonance. Evanston, IL: Row Peterson.

Gilovich, T., \& Medvec, V. H. (1995). The experience of regret: What, when, and why. Psychological Review, 102, 379-395. https://doi.org/10.1037/0033-295X.102.2.379

Giner-Sorolla, R. (2001). Guilty pleasures and grim necessities: Affective attitudes in dilemmas of self-control. Journal of Personality and Social Psychology, 80, 206-221. https://doi.org/10.1037/0022-3514.80.2.206

Godin, G. (2011). The Godin-Shephard leisure-time physical activity questionnaire. The Health \& Fitness Journal of Canada, 4, 18-22.

Hayes, J., Schimel, J., Arndt, J., \& Faucher, E. H. (2010). A theoretical and empirical review of the death-thought accessibility concept in terror management research. Psychological Bulletin, 136, 699-739. https://doi.org/10.1037/a0020524

Janis, I. L., \& Mann, L. (1977). Decision making: A psychological analysis of conflict, choice, and commitment. New York, NY, US: Free Press.

Kaiser, F. G., Byrka, K., \& Hartig, T. (2010). Reviving Campbell's paradigm for attitude research. Personality \& Social Psychology Review (Sage Publications Inc.), 14, 351-367. https://doi.org/10.1177/1088868310366452

Kaklamanou, D., Armitage, C. J., \& Jones, C. R. (2013). A further look into compensatory health beliefs. British Journal of Health Psychology, 18, 139-154. 
Kilpatrick, M., Hebert, E., \& Bartholomew, J. (2005) College Students' motivation for physical activity: Differentiating men's and women's motives for sport participation and exercise. Journal of American College Health, 54, 87-94. https://doi. org/10.3200/JACH.54.2.87-94

Knäuper, B., Rabiau, M., Cohen, O., \& Patriciu, N. (2004). Compensatory health beliefs: scale development and psychometric properties. Psychology\& Health, 19, 607624. https://doi.org/10.1080/0887044042000196737

Kronick, I., Auerbach, R. P., Stich, C., \& Knäuper, B. (2011). Compensatory beliefs and intentions contribute to the prediction of caloric intake in dieters. Appetite, 57, 435-438. https://doi.org/10.1016/j. appet.2011.05.306

Kronick, I., \& Knäuper, B. (2010). Temptations elicit compensatory intentions. Appetite, 54, 398-401. https://doi.org/10.1016/j.appet.2009.12.011

Miller, D. T., Turnbull, W., \& McFarland, C. (1989). When a coincidence is suspicious: The role of mental simulation. Journal of Personality and Social Psychology, 57, 581-589. https://doi.org/10.1037/00223514.57.4.581

Miquelon, P., Knäuper, B., \& Vallerand, R. J. (2012). Motivation and goal attainment. The role of compensatory beliefs. Appetite, 58, 608-615. https:// doi.org/10.1016/j.appet.2011.12.025

Nudelman, G., \& Shiloh, S. (2015). Mapping health behaviors: constructing and validating a common sense taxonomy of health behaviors. Social Science \& Medicine, 146, 1-10. https://doi.org/10.1016/j. socscimed.2015.10.004

O'Connor, R. C., \& Armitage, C. J. (2003). Theory of planned behaviour and parasuicide: An exploratory study. Current Psychology, 22, 196-205. https:// doi.org/10.1007/s12144-003-1016-4

Quisenberry, A. J., Eddy, C. R., Patterson, D. L., Franck, C. T., \& Bickel, W. K. (2015). Regret expression and social learning increases delay to sexual gratification. PLoS One, 10, e0135977. https://doi. org/10.1371/journal.pone.0135977

Rabiau, M., Knäuper, B., \& Miquelon, P. (2006). The eternal quest for optimal balance between maximizing pleasure and minimizing harm: The compensatory health beliefs model. British Journal of Health Psychology, 11, 139-153. https://doi. org/10.1348/135910705X52237

Rabiau, M. A., Knäuper, B., Nguyen, T. K., Sufrategui, M., \& Polychronakos, C. (2009). Compensatory beliefs about glucose testing are associated with low adherence to treatment and poor metabolic control in adolescents with type 1 diabetes. Health Education Research, 24, 890-896. https://doi.org/10.1093/her/ cyp032

Radtke, T., \& Scholz, U. (2016). Beliefs are not behavior. Zeitschrift für Gesundheitspsychologie, 24, 119-129.

Radtke, T., Scholz, U., Keller, R., \& Hornung, R. (2012). Smoking is ok as long as I eat healthily: Compen- satory Health Beliefs and their role for intentions and smoking within the Health Action Process Approach. Psychology \& Health, 27, 91-107.

Richard, R., de Vries, N. K., \& van der Pligt, J. (1998). Anticipated regret and precautionary sexual behaviour. Journal of Applied Social Psychology, 28, 1411-1428. https://doi.org/10.1111/j.1559-1816.1998.tb01684.x

Richard, R., van der Pligt, J., \& de Vries, N. (1996). Anticipated affect and behavioural choice. Basic and Applied Social Psychology, 18, 111-129. https://doi. org/10.1207/s15324834basp1802_1

Sandberg, T., \& Conner, M. (2008). Anticipated regret as an additional predictor in the theory of planned behaviour: A meta-analysis. British Journal of Social Psychology, 47, 589-606. https://doi. org/10.1348/014466607X258704

Sheeran, P., \& Orbell, S. (1999). Augmenting the theory of planned behaviour: Roles for anticipated regret and descriptive norms. Journal of Applied Social Psychology, 29, 2107-2142. https://doi. org/10.1111/j.1559-1816.1999.tb02298.x

Sherman, S. J. (1980). On the self-erasing nature of errors of prediction. Journal of Personality and Social Psychology, 39, 211-221. https://doi.org/10.1177/0022022106297301

Thompson, E. R. (2007). Development and validation of an internationally reliable short-form of the Positive and Negative Affect Schedule (PANAS). Journal of Cross-Cultural Psychology, 38, 227-242. https://doi.org/10.1177/0022022106297301

Zeelenberg, M., \& Pieters, R. (2007). A theory of regret regulation 1.0. Journal of Consumer Psychology, 17, 3-18.
Regret and compensatory behaviors 